\title{
Balancing Prediction and Sensory Input in Speech Comprehension: The Spatiotemporal Dynamics of Word Recognition in Context
}

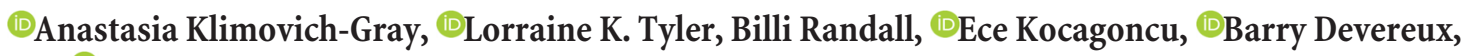 \\ and $\odot$ William D. Marslen-Wilson \\ Centre for Speech, Language and the Brain, Department of Psychology, University of Cambridge, Cambridge, CB2 3EB, United Kingdom
}

\begin{abstract}
Spoken word recognition in context is remarkably fast and accurate, with recognition times of $\sim 200 \mathrm{~ms}$, typically well before the end of the word. The neurocomputational mechanisms underlying these contextual effects are still poorly understood. This study combines source-localized electroencephalographic and magnetoencephalographic (EMEG) measures of real-time brain activity with multivariate representational similarity analysis to determine directly the timing and computational content of the processes evoked as spoken words are heard in context, and to evaluate the respective roles of bottom-up and predictive processing mechanisms in the integration of sensory and contextual constraints. Male and female human participants heard simple (modifier-noun) English phrases that varied in the degree of semantic constraint that the modifier (W1) exerted on the noun (W2), as in pairs, such as "yellow banana." We used gating tasks to generate estimates of the probabilistic predictions generated by these constraints as well as measures of their interaction with the bottom-up perceptual input for W2. Representation similarity analysis models of these measures were tested against electroencephalographic and magnetoencephalographic brain data across a bilateral fronto-temporo-parietal language network. Consistent with probabilistic predictive processing accounts, we found early activation of semantic constraints in frontal cortex (LBA45) as W1 was heard. The effects of these constraints (at $100 \mathrm{~ms}$ after W2 onset in left middle temporal gyrus and at $140 \mathrm{~ms}$ in left Heschl's gyrus) were only detectable, however, after the initial phonemes of W2 had been heard. Within an overall predictive processing framework, bottom-up sensory inputs are still required to achieve early and robust spoken word recognition in context.
\end{abstract}

Key words: language; prediction; RSA; speech; time-course; word recognition

Significance Statement

Human listeners recognize spoken words in natural speech contexts with remarkable speed and accuracy, often identifying a word well before all of it has been heard. In this study, we investigate the brain systems that support this important capacity, using neuroimaging techniques that can track real-time brain activity during speech comprehension. This makes it possible to locate the brain areas that generate predictions about upcoming words and to show how these expectations are integrated with the evidence provided by the speech being heard. We use the timing and localization of these effects to provide the most specific account to date of how the brain achieves an optimal balance between prediction and sensory input in the interpretation of spoken language.

\section{Introduction}

Processing spoken words involves the activation of multiple word candidates and competition between them until one candidate becomes uniquely compatible with the incremental speech input (Marslen-Wilson and Welsh, 1978; Zhuang et al., 2014; Koca-

Received Dec. 15, 2017; revised 0ct. 17, 2018; accepted 0ct. 18, 2018

Author contributions: L.K.T., B.R., E.K., and B.D. designed research; E.K. performed research; L.K.T., B.R., B.D., and W.D.M.-W. contributed unpublished reagents/analytic tools; A.K.-G. analyzed data; A.K.-G., L.K.T., and W.D.M.-W. wrote the paper.

This work was supported by a European Research Council Advanced Investigator Grant to L.K.T. under the European Community's Horizon 2020 Research and Innovation Programme (2014-2020 ERC Grant Agreement 669820), and Isaac Newton Trust Research Grant 2017 Grant 15.40(k) to L.K.T. goncu et al., 2017). In everyday speech, despite the low predictability of successive words in natural discourse (Luke and Christianson, 2016), this recognition process is strongly affected by the prior context, with recognition times of $\sim 200 \mathrm{~ms}$ from word onset, well before all of the word has been heard (Marslen-

Correspondence should be addressed to Dr. Lorraine K. Tyler, Centre for Speech, Language and the Brain, Department of Psychology, University of Cambridge, Downing Street, Cambridge CB2 3EB, United Kingdom. E-mail: Iktyler@csl.psychol.cam.ac.uk.

B.D. is now based at Queens University, Belfast, Northern Ireland.

https://doi.org/10.1523/JNEUROSCI.3573-17.2018

Copyright $\odot 2019$ the authors $\quad 0270-6474 / 19 / 390519-09 \$ 15.00 / 0$ 
Wilson, 1975; Marslen-Wilson and Tyler, 1975; Sereno et al., 2003). The neurocomputational mechanisms underlying these powerful and early contextual effects remain unclear and in dispute.

Early bottom-up models assigned a primary role to the speech input in generating an initial cohort of word candidates, with context affecting the selection of the unique word candidate from among this set (Tyler and Wessels, 1983; Marslen-Wilson, 1987). A range of other models allowed contextual constraints to modulate directly the state of potential word candidates before any of the word was heard (Morton, 1969; e.g., McClelland and Elman, 1986). More recently, the influential predictive coding framework views language comprehension as being driven by an internal generative model, which reduces uncertainty about perceptual interpretation by generating probabilistic top-down hypotheses about potential upcoming words (Friston and Frith, 2015; Kuperberg and Jaeger, 2016). These hypotheses, generated in frontal cortex (Sohoglu and Davis, 2016), result in preactivation of brain areas relevant to the processing of lexical form and content, so that top-down predictions can be compared with upcoming sensory data.

The strength and specificity of such predictions will vary as a function of preceding contextual constraints, with strong constraints generating lexically specific predictions and weaker constraints generating more graded semantic and syntactic predictions (Kuperberg and Jaeger, 2016; Luke and Christianson, 2016). To date, however, almost all research into the neural substrate for predictive processing has used constraining contexts where the target is highly predictable. It remains unclear specifically where and when predictive constraints apply at more natural levels of constraint, and how these relate to incoming constraints provided by the sensory input.

Here we use source-localized electroencephalographic and magnetoencephalographic (EMEG) data, combined with multivariate representation similarity analysis (RSA), to determine directly the timing and the neurocomputational content of the processes evoked as spoken words are heard in variably constraining contexts, reflecting the levels of predictability seen in natural discourse. Participants listened to two word phrases (e.g., "yellow banana"), which varied the specificity of the semantic constraint imposed by the modifier (W1) on the noun (W2). To express these constraints as probability distributions of potential word candidates, we used a gating task in which participants listened to incremental fragments of the word pairs and produced possible continuations (Grosjean, 1980). Based on these candidate sets, we devised several probes of the neural mechanisms whereby the constraints generated by hearing W1 could affect the processing of $\mathrm{W} 2$.

Three measures were based on the candidate sets generated when only W1 had been heard, asking whether different predictive representations of candidate properties were computed as W1 was heard, and how they modulated future processing events. An Entropy model captured the degree of uncertainty about the lexical identity of W2, given W1. A Semantic Similarity model captured the overall semantic dispersion of the predicted candidate sets, probing the representational framework in terms of which the Entropy measure was computed. Third, a Semantic Blend model measured variations in the specific semantic content of the W1 candidate sets. These variations can potentially affect semantically related processing activity in lexical representation areas, both before and after the onset of W2. A fourth model, Entropy Change, reflects the shift in the distribution of predicted W2 candidates given the early perceptual input from
W2. This measures the interaction between context-derived constraints and bottom-up perceptual input as W2 is heard. Using RSA, we evaluated these four models against EMEG sourcelocalized activity estimates within an extended bilateral frontotemporo-parietal language mask.

\section{Materials and Methods}

\section{Participants}

EMEG data acquisition was performed on 20 healthy participants ( 9 males) with a mean age of 23.8 years (range: 20-34 years). All were right-handed native British English speakers with normal hearing and normal or corrected-to-normal vision. The data for 4 subjects were discarded due to poor EEG quality. The experiment was approved by the Cambridge Psychology Research Ethics Committee.

\section{Stimuli}

The test stimuli consisted of 154 spoken English noun phrases where an adjective modifier (W1) was followed by a noun (W2). All nouns were concrete objects selected from the CSLB norms database (Devereux et al., 2014) with average word form frequency of 14.9 per million, $S D=18.3$ (CELEX) and mean noun duration of $605 \mathrm{~ms}, \mathrm{SD}=107 \mathrm{~ms}$. In contrast to previous studies which emphasized highly constraining contexts, here we aimed for a moderate overall degree of constraint between W1 and W2. The modifier adjectives were selected to vary the strength of the semantic constraint they exerted on the noun. Constraint strength varied from relatively weak (e.g., "yellow banana") to relatively strong (e.g., "peeled banana") and was quantified by using frequency information from Google ngrams (2007-2008, British English corpus). This allowed us to calculate the log-transformed conditional probability of a phrase ("yellow banana") given the modifier ("yellow") as follows:

$$
\text { Constraint }=-\log \left(P\left(C_{i j} \mid C_{i}\right)\right)=-\log \frac{P\left(C_{i} \cap C_{j}\right)}{P\left(C_{i}\right)}
$$

Here $C_{\mathrm{ij}}$ is the frequency of a given two-word phrase ("yellow banana") and $C_{\mathrm{i}}$ is the frequency of the modifier ("yellow") alone. The average constraint value for the test stimulus set was 1.89 (SD 1.06), corresponding to a non-log-transformed conditional probability average of 0.08 $(\mathrm{SD} 0.19$; minimum $=0$; maximum $=0.8)$. This level and range of transitional probability between the word pairs are similar to the results of parallel computations for larger corpora, including short narratives (e.g., Luke and Christianson, 2016). This confirms that the balance of lower and higher constraint in the present stimulus set is consistent with naturally occurring degrees of predictability between successive words.

A separate gating study with 35 participants was also conducted on these word pairs (as described in the next section), from which we could calculate the cloze probability of W2 given W1. This was estimated at 0.17 $(\mathrm{SD}=0.29)$, confirming that the probability of predicting the specific target word (W2) was generally low for this stimulus set.

To reduce the overall proportion of predictive modifiers in the set of word pairs heard by the participants, we also included 77 modifier-noun filler pairs in which the noun was preceded by an unrelated word so that the 2 words did not form a meaningful phrase (e.g., "lullaby banana"). These filler items were not analyzed. This manipulation brought down the relatedness proportion from $100 \%$ to a relatively high $67 \%$, so that the majority of W1 items were still predictive (generally quite weakly) of the properties of the following W2 (but see Delaney-Busch et al., 2017).

The presentation order of the phrases was pseudo-randomized and split into three blocks. To minimize the effects of block order, blocks were presented in 6 different orders across participants. The mean duration of phrases was $1213 \mathrm{~ms}$, and the duration of each block was $\sim 10 \mathrm{~min}$.

The stimuli were recorded onto digital audio tape at a sampling rate of $44,100 \mathrm{~Hz}$ by a female native speaker of British English. All word pairs were recorded as a single spoken phrase, with no added interval inserted between W1 onset and W2 offset. Recordings were transferred to computer and downsampled to 22,050 Hz, 16 bits, mono-channel format using Cool Edit Software (Syntrillium Software). 


\section{Experimental design and statistical analysis}

Behavioral pretesting and gating. A behavioral pretest was conducted to rate the naturalness of the test stimulus phrases. Twenty participants (mean age 24.8 years, 13 females), who did not participate in the MEG experiment, listened to the stimuli and rated the naturalness of each two word test phrase on a scale of $1-5(5=$ high naturalness $)$. The final set of 154 phrases contained only phrases judged to be highly natural $($ mean $=$ $4.32, \mathrm{SD}=0.42$ ).

We also ran a behavioral gating study (Grosjean, 1980) with a separate group of 35 participants (mean age 20.6 years, range $18-35$ years, 13 males) who were instructed (1) to listen to W1 followed by spoken segments of W2 presented incrementally in $50 \mathrm{~ms}$ intervals; (2) to type in their best guess of what that word might be after each segment; and (3) to rate their confidence (1-7 from least to most confident). We used responses at the offset of W1 (Gate 0) to derive measures of Entropy, Semantic Similarity, and Semantic Blend. Responses at later gates were used to estimate an Entropy Change model, as well the W2 identification point, defined as the average time (ms) from W2 onset when $80 \%$ participants produced the correct W2 response twice in a row (Grosjean, 1980; Tyler and Wessels, 1983). The mean identification point of W2 was $240 \mathrm{~ms}$ (SD $125 \mathrm{~ms}$ ) from word onset.

Cognitive models for RSA analyses. To determine how the probabilistic distribution of W2 candidates (derived from the gating experiment at the offset of W1) differed according to the strength of W1 constraints, and how these interacted with the processing of W2, we generated four cognitive models, as described below. The multivariate RSA technique then allows us to test for neural activity corresponding to these models against source-localized brain data covering an extended bilateral frontotemporo-parietal language mask.

\section{Entropy}

To capture the shape of the probability distribution of potential W2 candidates for each word pair, as estimated by gating data obtained at the offset of W1 (Gate 0), we calculated a single metric of Entropy, using Shannon's entropy $(\mathrm{H})$ formula as follows:

$$
H=-\sum_{i=1}^{n} P\left(x_{i}\right) \log P\left(x_{i}\right)
$$

Here $P\left(x_{\mathrm{i}}\right)$ is the summed confidence score for a given W2 competitor at Gate 0 across all participants divided by the sum of all confidence scores of all W2 competitors for that item across all participants.

High entropy indicates that there are many possible W2 candidates with low confidence scores because W1 only weakly constrains upcoming W2 responses. In contrast, low entropy indicates that most participants in the gating task selected only one or two specific word continuations, reflecting stronger W1 constraints on W2 for that word pair.

These computations of probability distributions are relevant to the predictive processing approach in two ways. First, this approach requires that such representations (viewed as hypotheses about the future properties of the sensory input) are computed incrementally as the input is interpreted, so that they can modulate expectations of the properties of this input at the relevant levels of neural description. Second, the pattern of variation in these probability distributions should modulate these patterns of neural activity before the onset of W2 as well as after. A bottom-up priority model, in contrast, predicts that such effects can only be seen after W2 onset, when an initial cohort has begun to be established.

Semantic similarity. The Entropy measure reflects the probability distribution of word candidates produced at the offset of W1 for each phrasal modifier. This measure, however, simply tabulates the number (and associated confidence) of the word candidates generated. It is likely, however, given the low average transitional probability of the modifiernoun pairs used here, that the words generated for most W1 modifiers reflected broad semantic and syntactic constraints on possible W2 continuations rather than specific hypotheses about lexical forms.

To measure the semantic distributional properties of these candidate sets, we calculated the pairwise similarity of the same sets of words, using a corpus-based Distributional Memory database (Baroni and Lenci, 2010). This represents words as vectors $>5000$ semantic dimensions, where these dimensions are distilled from word co-occurrence data. The Semantic Similarity of the candidates for each phrasal modifier was defined as the average pairwise cosine similarity between vectors for all its Gate 0 word candidates. The stronger the semantic constraint that the modifier places on the following word, the less semantic dispersion there will be in the set of candidate words that it evokes. After the modifier "cashmere," for example, gating participants tended to produce words from the semantic category "clothing" (e.g., "sweater," "scarf"). For a less constraining modifier like "massive," there was much more variability in the semantics of suggested words, "car," "tower," and "blow."

If the probability distributions for W2 candidates are well captured by the semantic dispersion between candidates, then model fit should parallel the results for the Entropy measure because they would both reflect the semantic constraints generated by the W1 modifier. Further, if the informational substrate for computing constraints is primarily semantic/ distributional in nature and represented in middle temporal cortices, then any top-down modulation involving frontal regions (e.g., Musz and Thompson-Schill, 2017) may primarily affect lexical semantic representations rather than phonological representations in superior temporal gyrus and Heschl's gyrus (HG) (Binder et al., 2000).

Semantic blend. Entropy and Semantic Similarity models capture the overall properties of the distribution of expected word candidates, the shape of the candidate distribution, and its dispersion in the semantic domain. However, they do not directly test the retrieval of the predicted candidate-specific semantic content of W2. To test when, with respect to W2 auditory onset, and in which brain regions such information is accessed, we derived the Semantic Blend model.

The Distributed Cohort Model of speech perception (Gaskell and Marslen-Wilson, 1997, 2002) assumes that lexico-semantic information associated with any given item involves a distributed activity pattern across the lexico-semantic representational space. When multiple word candidates are accessed, the resulting pattern of activation should reflect this ambiguity and encode a "blend" of overlapping representations, where lexico-semantic features shared across multiple candidates dominate the activation pattern. To derive Semantic Blends for each stimulus (e.g., "yellow banana"), we first normalized the semantic vectors (Baroni and Lenci, 2010) obtained from the gating responses collected at Gate 0. This was to prevent outlying values for a given candidate from dominating the blend. These normalized (by vector length) candidate vectors were averaged to produce a single "blend" vector, weighting each vector by its associated confidence score. The resulting blend therefore mixed together the semantics of every W2 candidate, with a more coherent blend reflecting greater degrees of W1 constraint. In terms of evaluating the balance between predictive and bottom-up inputs under the relatively weak levels of constraint present in this study, we ask here whether these variations in the predictability of W2 candidate semantics will be reflected before W2 onset in the patterns of neural activity in temporal lobe areas responsible for the representation of lexical semantics, or whether they will require the additional constraints provided by the initial phonemes of W2.

Entropy change. The above models capture potential predictive constraints that can be generated once W1 has been recognized and before W2 onset. To examine how these W1 constraints interact with the early perceptual input for W2, we subtracted the entropy values calculated at Gate 0 (as in the Entropy model) from the entropy values calculated from the candidates generated at Gate 1,50 ms after the onset of W2. At Gate 1 , the first phoneme of W2 will in most cases be identifiable, so that the candidates generated could potentially be constrained both by bottom-up phonological cues and by the constraints derived from W1. There was a significant drop ( $p<0.001$, paired $t$ test $)$ in Entropy at Gate 1 (mean 1.78), compared with Gate 0 (mean 2.14).

On a predictive processing approach, the bottom-up constraints available early in W2 should serve to update expectations about existing W2 lexical candidates, with corresponding reductions in lexical uncertainty. This in turn could lead to model fit in brain areas responsible both for computing candidate probabilities and for using these hypotheses to modulate incoming perceptual analyses. The timing of these activations 
is uncertain but may require more input than just the first $50 \mathrm{~ms}$ of the word in question.

\section{Procedure}

The auditory stimuli were delivered binaurally through MEGcompatible ER3A insert earphones (Etymotic Research). The instructions to the participants were visually presented on a monitor screen positioned $1 \mathrm{~m}$ in front of them. They were told to listen attentively to the word pairs. To encourage them to do so, on $10 \%$ of the trials, the spoken phrase (e.g., "school bus") was followed by a single written word in the middle of the screen (e.g., "children?"). Participants were instructed to judge the semantic compatibility of the written word with the preceding phase and to give a yes/no answer via button presses. These semantically related question trials were followed by a standard spoken phrase trial, which was treated as a dummy and not included in the analyses.

E-Prime Studio version 2 (Psychology Software Tools) was used to present the stimuli and record participants' responses. The experiment began with a short practice run, which was followed by three experimental blocks. The duration of the entire experiment was $\sim 40 \mathrm{~min}$. Before each spoken stimulus presentation, a cross appeared in the middle of the screen for $650 \mathrm{~ms}$ prompting the participant to focus their eyes on the cross. The intertrial interval, measured from the offset of the spoken phrase, was jittered between 3000 and $4000 \mathrm{~ms}$. The duration of the intertrial interval for the infrequent "question trials" was the same overall as for the standard trials. To reduce the potential contamination of the EMEG recordings by blink artifacts during the test phase, the last $1500 \mathrm{~ms}$ of each intertrial interval was made up of a "blink break," during which participants were encouraged to blink their eyes. The start of the blink break was indicated by an image of an eye that appeared in the middle of the screen. Participants were also asked to refrain from movement during the entire block of recording.

\section{EMEG recording}

Continuous MEG data were recorded in a magnetically shielded room (Imedco) at the MRC Cognition and Brain Sciences Unit in Cambridge, using the Vector View system (Elektra-Neuromag) containing 102 magnetometers and 204 planar gradiometers, arranged within a helmet covering the head of the subject. The position of the head relative to the sensors was monitored using the Head Position Indicator coils attached to the subject's head. EEG was recorded simultaneously from $70 \mathrm{Ag}-\mathrm{AgCl}$ electrodes within an elastic cap (Esacycap) on the subject's head. Vertical and horizontal EOGs were also recorded for blink detection. A 3D digitizer was used to record the position of the EEG electrodes and the Head Position Indicator coils and $\sim 100-150$ head points along the participants' scalp, relative to the three anatomical positions (the nasion and the left and right preauricular points). Acquired data were sampled at $1 \mathrm{kHz}$ and bandpass filtered from 0.03 to $330 \mathrm{~Hz}$.

\section{Data preprocessing}

The raw data were processed using MaxFilter 2.2 (Elekta). Static magnetometer and gradiometer bad channels were identified and reconstructed using interpolation. Temporal extension of the signal space separation technique (Taulu et al., 2005) was applied to separate the external noise from the head-internal signals. Finally, correction for head movement across the blocks was applied, and each subject's data were transformed to a default head position. The EEG raw data were then manually inspected and noisy EEG channels were removed.

The raw data were low pass filtered to $200 \mathrm{~Hz}$ and notch filtered at 50 $\mathrm{Hz}$ (to remove the mainline frequency components). Independent component analysis was applied to the MEG and EEG data separately for further denoising (minimum norm estimate [MNE] python) (Gramfort et al., 2013). The ICA components most strongly correlating with the vertical EOG channel were identified and removed. For the current analysis, raw data were then further bandpass filtered 0.1 to $40 \mathrm{~Hz}$, all previously identified bad channels were interpolated.

The data file for each test trial was then epoched with respect to the auditory onset of W2. The W2 onset epoch was $500 \mathrm{~ms}$ in length (range: -200 to $300 \mathrm{~ms}$, aligned to W2 auditory onset). During epoching, the data were baseline corrected using $100 \mathrm{~ms}$ of prestimulus data that did not contain any speech signal. Trials with large artifacts were considered noisy and were removed (EEG $>150 \mu \mathrm{V}$, magnetometer $>5 \mathrm{e}-12 \mathrm{~T}$ gradiometer $>2000 \mathrm{e}-13 \mathrm{~T} / \mathrm{cm})$.

\section{Source reconstruction}

The preprocessed data were source localized by using the MNE procedure (MNE Python) (Gramfort et al., 2013) based on distributed source modeling (Lin et al., 2006). MNE constrains the sources of currents by using a priori assumptions about their distributions (dipole orientation and location summarized in the lead field matrix, derived from the structural MRI scans) and the noise estimates covariance matrix.

The first step in source localization was the acquisition of structural MRI images of each participant using the GRAPPA 3D MPRAGE sequence (time repetition $=2250 \mathrm{~ms}$; time echo $=2.99 \mathrm{~ms}$; flip angle $=9$; acceleration factor $=2$ ) on a 3 -T Trio scanner (Siemens) with $1 \mathrm{~mm}$ isotropic voxels. MRI structural images were processed with FreeSurfer software (Fischl, 2012) to parcellate brain volumes into inner and outer skin and skull, white and gray matter. Subsequent steps were performed using the MNE Python environment. The source grid was set up on the white-gray matter boundary surface and downsampled to 4098 sources per hemisphere. The MRI and MEG coordinate systems were coregistered using the MNE analysis interface, with respect to the anatomical locations marked during acquisition (the nasion and the left and right preauricular points) and additional $100-150$ head points.

Second, for each subject, the forward model (lead field matrix) was created using a 3-layer boundary element model that assigned different electrical conductivities to inner and outer surfaces (5120 triangles per surface) of the skull. A regularized covariance matrix was estimated from the epochs using the baseline period. The forward solution and the covariance matrix were used to estimate the linear inverse regularization parameter (inverse operator) for every source across all channels. To improve the spatial accuracy of the localization and correct for a bias toward assigning signals to superficial sources, a loose source orientation (0.2) constraint and a depth constraint (0.8) were applied (Lin et al., 2006). To derive the source estimates at every time point and for every trial, the inverse operator was applied to preprocessed data by taking the norm of the dipole components. The estimated activations were normalized with respect to signal noise by dividing the estimates by their predicted SE, thus producing unsigned dynamic statistical parametric maps (Dale et al., 2000). To account for increased noise levels in the single-trial estimates, the $\lambda^{2}$ parameter was set to 1 . The subject-specific estimates were then morphed to the averaged brain surface (produced with FreeSurfer) for further statistical analysis.

\section{$R S A$}

RSA makes it possible to compare directly the correlational structure in patterns of brain activity with the correlational structures predicted by different cognitive models (Kriegeskorte et al., 2008). We used a sliding window RSA, in which each subject's data Representational Dissimilarity Matrices (RDMs) across the length of the epoch were compared with cognitive model RDMs within every ROI (for a schematic overview of the RSA and parameters used, see Fig. 1). Distinct cognitive model RDMs were derived for each cognitive measure (matrix size $154 \times 154$, reflecting the number of test phrases). The Entropy RDM was defined by taking the absolute pairwise differences between the lexical Entropy scores for all word pairs. The Semantic Similarity model RDM was produced by taking the absolute pairwise differences of the Semantic Similarity scores associated with each word pair. The Semantic Blend model RDM was computed by taking the pairwise cosine distances between every pair of "blended" semantic vectors (of Gate 0 predicted candidates for each word pair). The Entropy Change RDM was calculated by taking the absolute pairwise differences in the Entropy Change measure. Grouplevel data were derived by extracting subject-specific model-fit $r$ values and conducting a one-sample $t$ test across subjects at each ROI and time point. Only $t$ value clusters that survived this initial $p<0.01$ threshold and then the correction for multiple comparisons (cluster-permutation permutation analysis) (Maris and Oostenveld, 2007; Su et al., 2012) at $p<0.05$ threshold are reported. We did not apply an additional correction for the number of ROIs tested. 


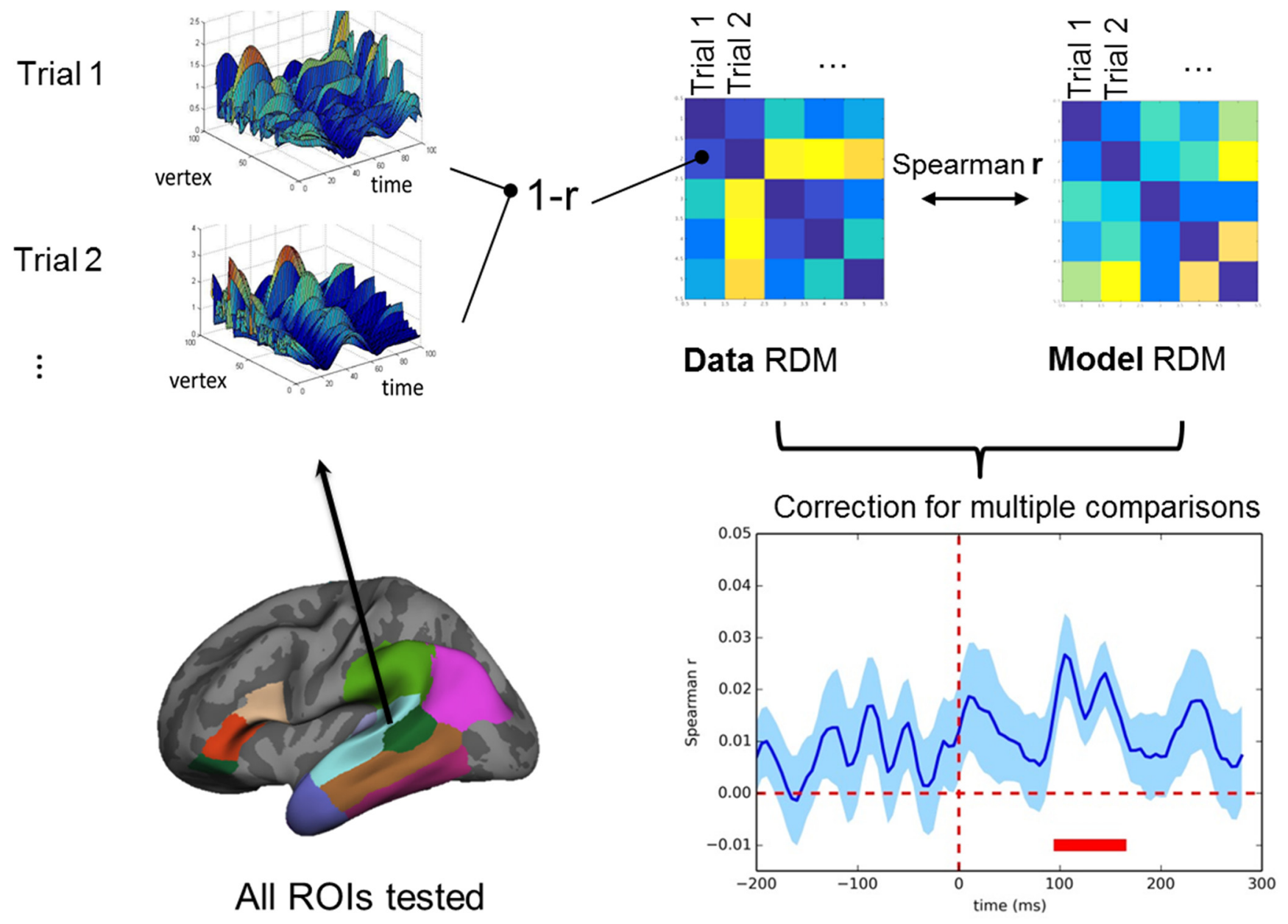

Figure 1. Schematic overview of RSA multivariate analysis procedures. (1) RDMs are derived for each ROl and each subject across the length of the analysis epoch. These data RDMs summarize the differences between the activation patterns $(1-r)$ between trials in a given time window ( $20 \mathrm{~ms}$ in width) every $5 \mathrm{~ms}$ across all vertices of a given ROI. (2) Each data RDM is compared with model RDMs (Spearman $r$ ), derived separately for each cognitive measure. To produce group-level statistics, a one-sample t test is taken for each ROl and each time point across subjects. The resulting $t$ maps were thresholded at the $p<0.01$ level. To correct for multiple comparisons, values surviving the primary threshold were entered into a cluster-permutation analysis (1000 permutations) (Maris and Oostenveld, 2007; Su et al., 2012). Only clusters that survived the $p<0.05$ cluster-correction threshold are reported (red bar).

The cognitive models outlined above (converted into model RDMs) were tested against activity patterns (captured in subjectwise data RDMs) in a set of bilateral frontotemporal ROIs (Fig. 1, inset, brain, ROIs taken from the Desikan-Killiany Atlas, Freesurfer) consisting of BA44, BA45, BA47, HG, posterior superior temporal sulcus, supramarginal gyrus, inferior-parietal area (which included the angular gyrus), and temporal pole. Temporal ROIs, superior temporal gyrus, middle temporal gyrus (MTG), and inferior temporal gyrus were split into mid-posterior and anterior parts, and the latter joined to form a functional anterior temporal lobe ROI. These ROIs were selected on the basis of previous studies showing their involvement in processing spoken language (Hickok and Poeppel, 2007; Hagoort, 2013; Tyler et al., 2013; Kocagoncu et al., 2017).

Finally, to assess the independence of the model-fit results for each RSA model from the other three models, we ran a set of additional partial correlation analyses. For each model separately, the model fit $(r)$ was estimated after a partial correlation analysis where the contribution of the other relevant models was partialled out. The rest of the procedure was identical to the main ROI RSA described above. The results of these analyses are reported alongside the results for each model tested separately.

\section{Results}

The questions at issue here concern the role of the constraints generated by $\mathrm{W} 1$ in the perception and identification of $\mathrm{W} 2$ : Whether or not the context (the W1 modifier) triggers access to a distribution of potential word (W2) candidates, what is the timing of this activation relative to $\mathrm{W} 1$ processing, whether and when this information affects the processing of W2, and how it interacts with the bottom-up constraints made available as W2 is heard. Three models, Entropy, Semantic Similarity, and Semantic Blend, encoded different properties associated with the probabilistic distribution of W2 candidates elicited at W1 offset, whereas the Entropy Change model tested for potential interactions between top-down contextual constraints and the early analysis of the perceptual input for W2 (for details, see Cognitive models). For all four models, the analyses were aligned to W2 onset, looking for potential model fit both before and after the start of the critical word (Fig. 2). While the average Identification Point for W2 (as estimated from the gating data) falls $240 \mathrm{~ms}$ after W2 onset, we expect to see much earlier effects on model fit as lexical candidates begin to be selectively activated by the incoming speech.

For all of the models reported below, the size of the model-fit $r$ values falls within the range of values obtained in comparable RSA analyses reported previously (Nili et al., 2014; e.g., Devereux et al., 2018).

The Entropy model (Fig. 2A) showed significant model fit in left BA45, starting $-70 \mathrm{~ms}$ before W1 offset and persisting until 
A

\section{LH BA 45}

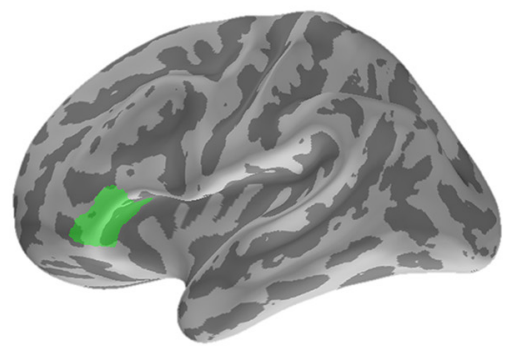

B

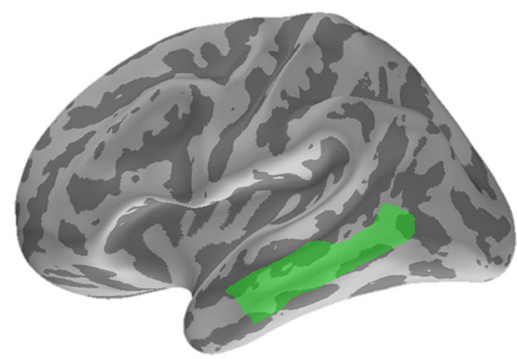

C

\section{LH HG}

D

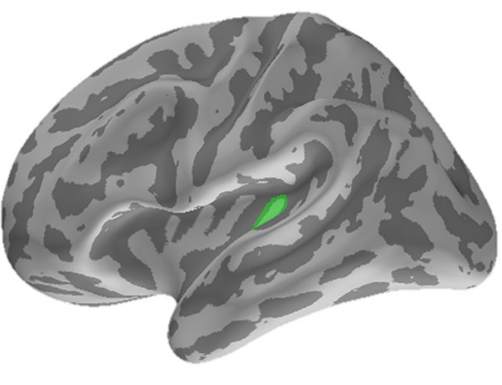

Figure Key

Mean W2 IP $=240 \mathrm{~ms}, \mathrm{SD}=125$

Mean W1 UP = $-193 \mathrm{~ms}, \mathrm{SD}=103$
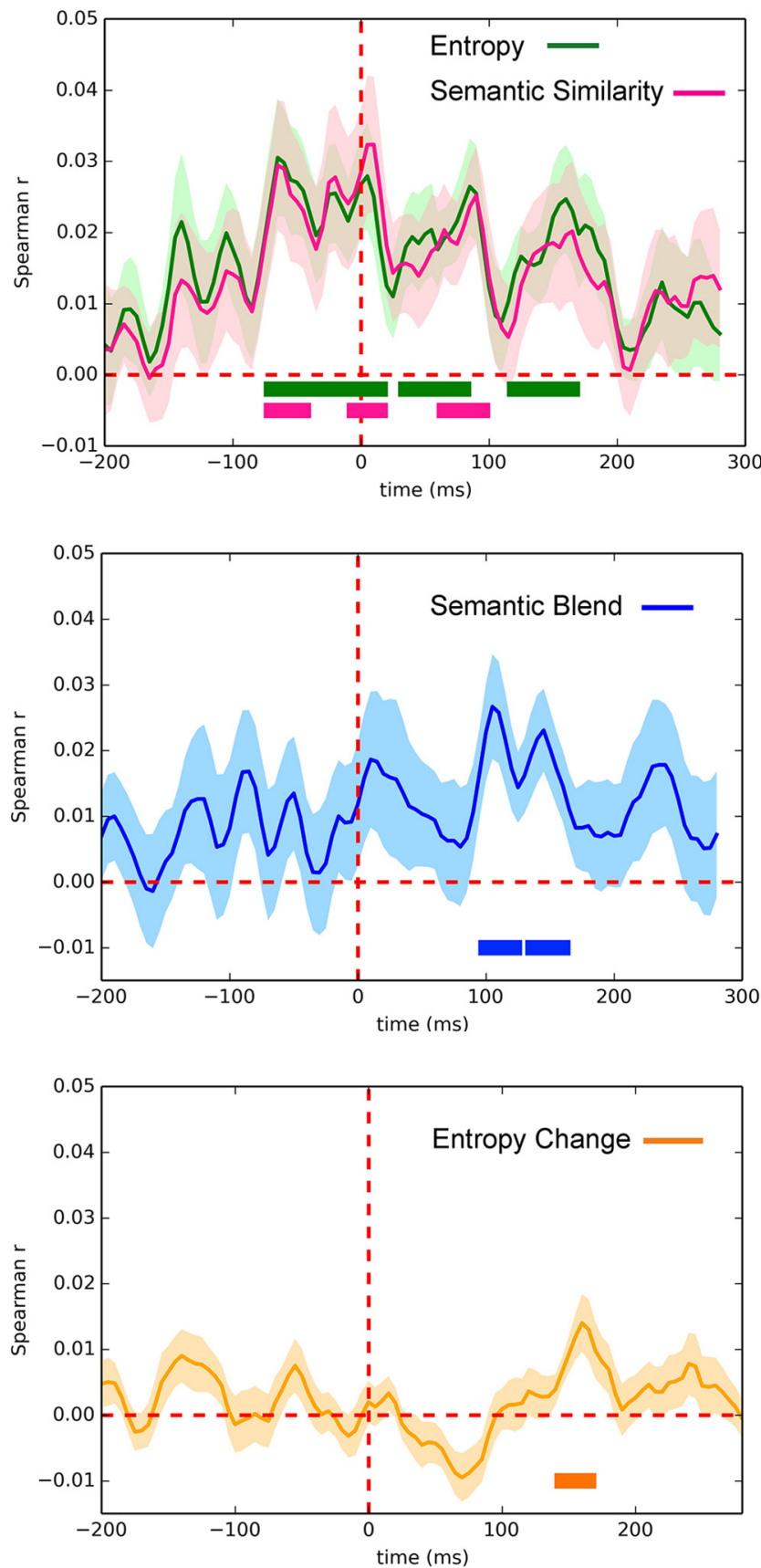

'corduroy trousers'

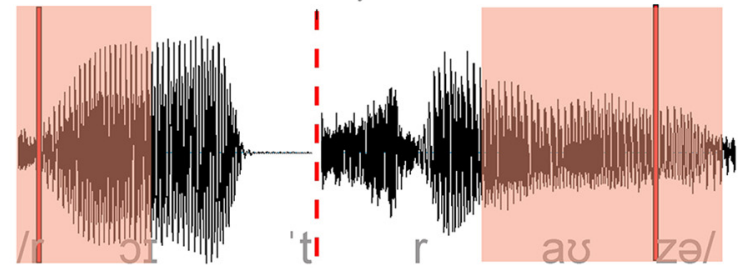

Mean W1 UP

W2 Onset

Mean W2 IP

Figure 2. Spatiotemporal coordinates of RSA model fits for the W1 modifier-based constraints. A-C, Left, The ROIs (in green on the inflated MNE brain) producing significant model fit for the four models. A, Right, Subject-averaged model fit $r$ value in left BA45 across the analysis epoch ( $-200 \mathrm{~ms}$ to $300 \mathrm{~ms}$ relative to W2 onset) for the Entropy (green) and the Semantic Similarity (pink) models. B, Semantic Blend (blue) model fit in left middle and posterior MTG. C, Entropy Change (orange) model fit in left HG. Shaded areas represent the SE of the model-fit means. Thick lines below the $r$ curves indicate the significant $t$ value model-fit clusters (corrected for multiple comparisons at $p<0.05$ ). Vertical broken red line indicates W2 onset. $\boldsymbol{D}$, Left, Key specifying the mean and SD of the critical time points overlaid (right) on the auditory waveform of a sample test phrase. 
165 ms after W2 onset: ( 1$)$ from $-70 \mathrm{~ms}$ to $15 \mathrm{~ms}(p<0.001)$; (2) from 35 to $80 \mathrm{~ms}(p<0.001)$; and (3) from $120 \mathrm{~ms}$ to $165 \mathrm{~ms}$ $(p=0.007)$. The Semantic Similarity model (Fig. $2 A)$ closely paralleled the Entropy model, also showing model fit only in left BA45, and also beginning at $-70 \mathrm{~ms}$ before W1 offset though finishing slightly earlier at $95 \mathrm{~ms}$ after W2 onset. The significant clusters were as follows: (1) from $-70 \mathrm{~ms}$ to $-45 \mathrm{~ms}(p=0.02)$; (2) from $-5 \mathrm{~ms}$ to $15 \mathrm{~ms}(p=0.03)$; and (3) from $65 \mathrm{~ms}$ to $95 \mathrm{~ms}$ $(p=0.01)$. The significant fit for both of these models starts after the W1 Identification Point (on average, 194 ms before W2 onset) and ends before W2 is recognized (on average, $240 \mathrm{~ms}$ after W2 onset). These results show that the number and probability (Entropy model) and the semantic dispersion (Semantic Similarity) of the W2 candidate distributions corresponded to patterns of neural activity that were activated after the modifier word had been recognized but before the onset of $\mathrm{W} 2$, and remained relevant to left inferior frontal processes well into the perceptual analysis of W2.

The close parallels between the timing and the location of the model fits elicited by the Entropy and Semantic Similarity models are consistent with the strong correlation (0.79) between their respective model RDMs, and with the claim that they both derive from the same underlying probabilistic source. The results of the partial correlation analysis confirm that the model fits for Entropy and Semantic Similarity are not independent, model fit in BA45 disappears across the board if Entropy is partialled out when estimating the effects of Semantic Similarity (and conversely for Semantic Similarity when Entropy is partialled out).

Turning to the Semantic Blend model, which tested for the processing relevance of the semantic content of the W2 candidates, we see a markedly different spatiotemporal pattern of model fit (Fig. 2B). No effects were found before W2 onset, and only left temporal locations were significant, with model fit in left middle and posterior MTG (Fig. 2B). The model fit was significant from 100 to $160 \mathrm{~ms}$ after W2 onset; initially from 100 to 120 $\mathrm{ms}(p=0.02)$ and then from 130 to $160 \mathrm{~ms}(p=0.03)$. These results show that semantic information about potential W2 candidates was accessed in MTG soon after the onset of W2 and before the word was uniquely identified. Furthermore, and consistent with the weak correlations between the Semantic Blend model RDM and the other cognitive model RDMs, the model fit remains significant in the partial correlation analysis with unchanged locations and almost identical time courses $(p=0.04$ for a first cluster from 100 to $115 \mathrm{~ms}$ and $p=0.02$ for a second cluster from 130 to $155 \mathrm{~ms}$ ) after partialling out the other three cognitive models. This implies that the Semantic Blend model captures processes distinct from those of Entropy and Semantic Similarity.

Fourth, the Entropy Change model (Fig. 2C), which captures the update in the W1 Entropy model after $50 \mathrm{~ms}$ of W2 perceptual input had been heard, produced significant model fit in left HG, from 140 to $180 \mathrm{~ms}(p=0.025)$ after the perceptual onset of W2. These effects again proved to be independent from the other three models, as confirmed through partial correlation analysis where a similar significant cluster $(p=0.035)$ was observed between 145 and 165 ms after W2 onset, consistent with the weak correlations between the Entropy Change model RDM and the other cognitive models.

A different set of partial correlation analyses were conducted to test the robustness of the model fit observed after W2 onset for the three "prediction" models (Entropy, Semantic Similarity, and Semantic Blend) against potentially uncontrolled variation in the bottom-up phonological and semantic properties of the W2 stimulus set. To address this issue, two additional models were constructed. To capture W2 semantics, we calculated pairwise cosine distances between the semantic vectors (Baroni and Lenci, 2010) for the W2 targets (as in Kocagoncu et al., 2017). For a phonological model, we converted the first two phonemes of each W2 into a binary articulatory feature vector (Wingfield et al., 2017), calculating pairwise cosine distances between each such vector to derive the model RDM. Separate partial correlation analyses were run for each cognitive model using the W2 semantic and phonological RDMs separately (the Entropy Change model was not included because this was already based on the integration of W1 predictions and bottom-up W2 constraints). For the critical period of interest from the onset of W2, we see no evidence that the timing and significance of model fit for the cognitive models expressing constraints derived from $\mathrm{W} 1$ are significantly confounded with W2 stimulus properties. There is no general weakening of the significance of the model fits obtained, nor is there any change in the basic timing of the relevant effects in relation to W2 onset for the three models.

Finally, to provide more fine-grained information about the spatiotemporal distribution of the model fit for each cognitive model, we also conducted searchlight analyses across the entire language mask bilaterally. The results of these exploratory analyses confirm that the ROI analyses reported in Figure 2 correctly identified the time periods and locations exhibiting consistent model fit for each model. No other spatially and temporally consistent clusters were visible in the searchlight data.

\section{Discussion}

This study addresses the specific neurobiological processes that underpin the dynamic and early integration of top-down and bottom-up constraints in the perceptual interpretation of spoken words heard in constraining contexts. We investigated these processes for a set of spoken two-word English phrases, presented in a minimal task "attentive listening" environment, and sampling a naturalistic range of degrees of constraint. The average predictability of the second word in the phrase was low, so that the available constraints were in general lexically nonspecific and broadly semantic in nature.

Using gating data, we generated four computational measures that captured specific properties of the probabilistic distribution of potential word candidates, where the combination of spatiotemporally well-resolved EMEG measures of dynamic brain activity, together with RSA multivariate techniques, made it possible to use these models to probe the neurocomputational content of processing activity as spoken words are heard. This provides a novel and revealing perspective on the dynamic functional architecture underpinning the integration of contextual prediction and sensory constraint in speech comprehension.

Overall, the results suggest a system for dynamically combining bottom-up and top-down constraints in speech interpretation that shares key characteristics of predictive processing with a strong dependence on the incoming speech input, necessarily so under conditions of weak contextual constraint. Consistent with the predictive processing approach, as soon as listeners recognized the W1 context word, they began to generate estimates of the probability distributions associated with potential W2 candidates. Significant RSA model fit for both Entropy and Semantic Similarity models was seen 70 ms before W2 onset (Fig. 2A).

These probability estimates, viewed as potential hypotheses about the upcoming word (W2), have several notable properties. The first is that model fit was seen only in left BA45 and not in any of the potential target regions for top-down modulation, such as auditory processing areas (HG and superior temporal gyrus) or 
lexical content regions in posterior and middle MTG. Although the processing role of BA45 is not fully understood, it is widely thought to be involved in semantic control processes (Novick et al., 2005; Musz and Thompson-Schill, 2017), and provides a plausible substrate for the computation of probabilistic semantic constraints (as indicated by the joint Entropy and Semantic Similarity model fit). We found no evidence, however, that the variations in constraint per se across word pairs being computed in BA45 were directly modulating neural patterns elsewhere in the language system.

A further significant property of the BA45 model fit is that it persisted well into the processing of W2, only dropping below significance at $165 \mathrm{~ms}$ after W2 onset for the Entropy model, and at $95 \mathrm{~ms}$ for the Semantic Similarity model. The probability estimates for W2 possible candidates based on W1 constraints, as computed by BA45, were apparently not modulated by bottom-up information about actual W2 candidates until at least the first two phonemes of W2 had been identified. In a predictive processing framework, where top-down hypotheses modulate the perceptual interpretation of the incoming sensory input, any mismatch between hypothesis and input is fed back, in the form of prediction error, to refine top-down hypothesis formation. The relative lateness with which $\mathrm{W} 1$ hypothesis sets continue to fit patterns of neural activity in BA45, together with the evidence that BA45 is operating in semantic terms, leads to the inference that the required feedback to BA45 is in terms of the semantic properties of actual W2 candidates and that the availability of this information depends on bottom-up cues to the identity of these candidates.

The claim that the operations of BA45 are primarily semantic in nature is consistent with the observed close similarity between the Entropy and Semantic Similarity models, not only in the model fit they exhibit over time (Fig. 2A), but also the close relationship of their respective RDMs in the partial correlation and cross-correlation measures. However, this lack of independence of the two models means that further research is still needed to confirm their specific shared underlying properties.

Supporting evidence for the role of W2 sensory input in enhancing access to candidate semantics comes from the Semantic Blend model (Fig. 2B), which captures variations in the semantic coherence of the sets of W2 candidates generated at Gate 0 . This shows a very different pattern of model fit to the Entropy and Semantic similarity models. There is no model fit before W2 onset, and none is seen until $100 \mathrm{~ms}$ into W2. The localization of this to left middle and posterior MTG is consistent with evidence that these regions support the processing of lexical semantic information (Binder et al., 2000; Hickok and Poeppel, 2007; Hagoort, 2013) but begs the question of why these regions only generate model fit well after W2 onset.

The timing and location of the Semantic Blend model fit make several points. First, the significant fit for predicted W2 semantics in left MTG is indeed likely to reflect an interaction between semantic hypotheses generated in BA45 and the processing of potential lexical candidates in relevant brain regions. However, this interaction only becomes neurocomputationally visible at $100 \mathrm{~ms}$ after W2 onset, once the bottom-up input has started to generate lexical candidates whose semantic properties overlap with those of W1-generated constraints. This also shows that the bottom-up activation of lexical contents occurs very early in the word, as early as access to lexical form (Marslen-Wilson, 1975; Kocagoncu et al., 2017). Third, the interaction is short-lived, terminating at $160 \mathrm{~ms}$ after onset, suggesting that the probabilistic constraints provided by $\mathrm{W} 1$ are rapidly superseded by new information from W2. The timing here is consistent with the termi- nation of model fit at $165 \mathrm{~ms}$ in BA45 for the W1 Entropy model, similarly displaced by new W2-based constraints.

Finally, the results for the Entropy Change model provide further evidence for the timing and the consequences of the interaction between bottom-up and contextual constraint. This model captures the reduction in lexical uncertainty generated by the integration of $\mathrm{W} 1$ constraints with the constraints provided by the initial $50 \mathrm{~ms}$ of W2. The resulting model fit is seen, with some delay at $140 \mathrm{~ms}$ after W2 onset, and is located in left HG, a brain region that supports the bottom-up perceptual analysis of the auditory input (Scott and Johnsrude, 2003; Uppenkamp et al., 2006; Warrier et al., 2009). The timing of this effect matches the time course indicated by the Semantic Blend results for the integration of semantic and phonological constraints, and suggests that convergence on specific word candidates is largely complete within 150-200 ms of word onset, consistent with behavioral evidence for the timing of word recognition in context (Marslen-Wilson, 1973, 1975). The location of the Entropy Change model fit in auditory cortex suggests, finally, that the interaction of bottom-up phonological cues with contextual semantic constraints is able to modulate activity in primary sensory processing regions as candidate words are heard, consistent with predictive processing claims (Sohoglu and Davis, 2016). However, at the generally weaker levels of constraint provided by the word pairs used here, the role of bottom-up perceptual evidence seems critical.

In conclusion, research into the neural substrate for predictive processing in language comprehension has emphasized stimulus sequences where predictive constraints are strong and lexically specific, and delivered in "prediction-friendly" experimental situations. In the current study, listeners heard words in less constraining contexts, arguably more representative of everyday language. In this situation, an "all-or-nothing" predictive processing regimen cannot hold, where contextual constraints predict the actual sensory forms of future inputs. Our word pairs were rarely sufficiently constrained to support such predictions.

We see instead a neurocognitive system where the basic components of a predictive processing framework are present, but where its forward predictions are tailored to the types of constraint that are at hand (largely semantic), and where bottom-up constraints are essential to the formation of phonologically and semantically specific perceptual hypotheses. There is evidence for early computation of probabilistic constraint representations, well before W2 onset, but we see no corresponding evidence for early modulation of neural activity in brain regions relevant to lexical form and content. It is only after information about the initial phonetic properties of W2 becomes available that we can detect interactions between W1 constraints and W2 interpretation, at $100 \mathrm{~ms}$ for the Semantic Blend model and at $140 \mathrm{~ms}$ for the form-specific predictions picked up by the Entropy Change model. The timing of the integration of these contextual and sensory constraints during W2 processing, while consistent with the rapid recognition of words in context, demonstrates that the sequential statistical properties of natural language require continuous contact with the sensory input to achieve the robust earliness of human real-time speech interpretation.

\section{References}

Baroni M, Lenci A (2010) Distributional memory: a general framework for corpus-based semantics. Comput Linguist 36:673-721. CrossRef

Binder JR, Frost JA, Hammeke TA, Bellgowan PS, Springer JA, Kaufman JN, Possing ET (2000) Human temporal lobe activation by speech and nonspeech sounds. Cereb Cortex 10:512-528. CrossRef Medline

Dale AM, Liu AK, Fischl BR, Buckner RL, Belliveau JW, Lewine JD, Halgren E 
(2000) Dynamic statistical parametric mapping. Neuron 26:55-67. CrossRef Medline

Delaney-Busch N, Morgan E, Lau E, Kuperberg GR (2017) Comprehenders rationally adapt semantic predictions to the statistics of the local environment: a Bayesian model of trial-by-trial N400 amplitudes. 39th Annual Conference of the Cognitive Science Society, London.

Devereux BJ, Tyler LK, Geertzen J, Randall B (2014) The Centre for Speech, Language and the Brain (CSLB) concept property norms. Behav Res Methods 46:1119-1127. CrossRef Medline

Fischl B (2012) FreeSurfer. Neuroimage 62:774-781. CrossRef Medline

Friston KJ, Frith CD (2015) Active inference, communication and hermeneutics. Cortex 68:129-143. CrossRef Medline

Gaskell MG, Marslen-Wilson WD (1997) Integrating form and meaning: a distributed model of speech perception. Lang Cogn Process 12:613-656. CrossRef

Gaskell MG, Marslen-Wilson WD (2002) Representation and competition in the perception of spoken words. Cogn Psychol 45:220-266. CrossRef Medline

Gramfort A, Luessi M, Larson E, Engemann DA, Strohmeier D, Brodbeck C, Hämäläinen M (2013) MEG and EEG data analysis with MNE-python. Front Neurosci 7:267. CrossRef Medline

Grosjean F (1980) Spoken word recognition processes and the gating paradigm. Percept Psychophys 28:267-283. CrossRef Medline

Hagoort P (2013) MUC (Memory, Unification, Control) and beyond. Front Psychol 4:416. CrossRef Medline

Hickok G, Poeppel D (2007) The cortical organization of speech processing. Nat Neurosci 8:393-402. CrossRef Medline

Kocagoncu E, Clarke A, Devereux BJ, Tyler LK (2017) Decoding the cortical dynamics of sound-meaning mapping. J Neurosci 37:1312-1319. CrossRef Medline

Kriegeskorte N, Mur M, Bandettini P (2008) Representational similarity analysis: connecting the branches of systems neuroscience. Front Syst Neurosci 2:4. CrossRef Medline

Kuperberg GR, Jaeger TF (2016) What do we mean by prediction in language comprehension? Lang Cogn Neurosci 31:32-59. CrossRef Medline

Lin FH, Witzel T, Ahlfors SP, Stufflebeam SM, Belliveau JW, Hämäläinen MS (2006) Assessing and improving the spatial accuracy in MEG source localization by depth-weighted minimum-norm estimates. Neuroimage 31: 160-171. CrossRef Medline

Luke SG, Christianson K (2016) Limits on lexical prediction during reading. Cogn Psychol 88:22-60. CrossRef Medline

Maris E, Oostenveld R (2007) Nonparametric statistical testing of EEG- and MEG-data. J Neurosci Methods 164:177-190. CrossRef Medline

Marslen-Wilson WD (1973) Linguistic structure and speech shadowing at very short latencies. Nature 244:522-523. CrossRef Medline

Marslen-Wilson WD (1975) Sentence perception as an interactive parallel process. Science 189:226-228. CrossRef Medline

Marslen-Wilson WD (1987) Functional parallelism in spoken word recognition. Cognition 25:71-102. CrossRef Medline

Marslen-Wilson WD, Tyler LK (1975) Processing structure of sentence perception. Nature 257:784-786. CrossRef Medline
Marslen-Wilson WD, Welsh A (1978) Processing interactions and lexical access during word recognition in continuous speech. Cogn Psychol 10: 29-63. CrossRef

McClelland JL, Elman JL (1986) The TRACE model of speech perception. Cogn Psychol 18:1-86. CrossRef Medline

Morton J (1969) Interaction of information in word recognition. Psychol Rev 76:165-178. CrossRef

Musz E, Thompson-Schill SL (2017) Tracking competition and cognitive control during language comprehension with multi-voxel pattern analysis. Brain Lang 165:21-32. CrossRef Medline

Nili H, Wingfield C, Walther A, Su L, Marslen-Wilson WD, Kriegeskorte N (2014) A toolbox for representational similarity analysis. PLoS Comput Biol 10:e1003553. CrossRef Medline

Novick JM, Trueswell JC, Thompson-Schill SL (2005) Cognitive control and parsing: reexamining the role of Broca's area in sentence comprehension. Cogn Affect Behav Neurosci 5:263-281. CrossRef Medline

Scott SK, Johnsrude IS (2003) The neuroanatomical and functional organization of speech perception. Trends Neurosci 26:100-107. CrossRef Medline

Sereno SC, Brewer CC, O'Donnell PJ (2003) Context effects in word recognition: evidence for early interactive processing. Psychol Sci 14:328-333. CrossRef Medline

Sohoglu E, Davis MH (2016) Perceptual learning of degraded speech by minimizing prediction error. Proc Natl Acad Sci U S A 113:1747-1756. CrossRef Medline

Su L, Fonteneau E, Marslen-Wilson WD, Kriegeskorte N (2012) Spatiotemporal searchlight representational similarity analysis in EMEG source space. International Workshop on Pattern Recognition in NeuroImaging, pp 97-100. University College London, London, UK.

Taulu S, Simola J, Kajola M (2005) Applications of the signal space separation method. Signal Processing 53:3359-3372.

Tyler LK, Wessels J (1983) Quantifying contextual contributions to word recognition processes. Percept Psychophys 34:409-420. CrossRef Medline

Tyler LK, Cheung TP, Devereux BJ, Clarke A (2013) Syntactic computations in the language network: characterizing dynamic network properties using representational similarity analysis. Front Psychol 4:1-19. CrossRef Medline

Uppenkamp S, Johnsrude IS, Norris D, Marslen-Wilson WD, Patterson RD (2006) Locating the initial stages of speech-sound processing in human temporal cortex. Neuroimage 31:1284-1296. CrossRef Medline

Warrier C, Wong P, Penhune V, Zatorre R, Parrish T, Abrams D, Kraus N (2009) Relating structure to function: Heschl's gyrus and acoustic processing. J Neurosci 29:61-69. CrossRef Medline

Wingfield C, Su L, Liu X, Zhang C,Woodland P, Thwaites A, Fonteneau E, Marslen-Wilson WD (2017) Relating dynamic brain states to dynamic machine states: Human and machine solutions to the speech recognition problem. PLoS Comput Biol 13(9):e1005617. CrossRef Medline

Zhuang J, Tyler LK, Randall B, Stamatakis EA, Marslen-Wilson WD (2014) Optimally efficient neural systems for processing spoken language. Cereb Cortex 24:908-918. CrossRef Medline 